\title{
Frequency, Risk Factors and Antibiotic Sensitivity Pattern of Extended-Spectrum Beta-Lactamase Producing Escherichia coli and Klebsiella pneumoniae Causing Urinary Tract Infection: Experience from a Tertiary Care Hospital of Bangladesh
}

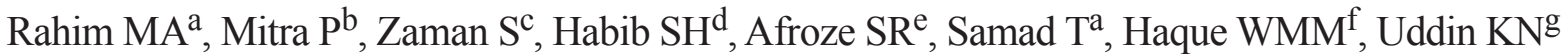

\begin{abstract}
Background: Urinary tract infection (UTI) due to extended-spectrum beta-lactamase (ESBL) producing organisms are increasing. This study was designed to evaluate the frequency of ESBL-positive Escherichia coli and Klebsiella pneumoniae causing UTI, their antibiotic sensitivity pattern and possible risk factors.

Methods: This case-control study was done in BIRDEM General Hospital, Dhaka, Bangladesh from January to March 2016. Patients with UTI due to ESBL-positive E. coli and K. pneumoniae were cases and non-ESBL organisms were controls.

Results: Total 98 patients with UTI due to E. coli (84) and K. pneumoniae (14) were eligible for analysis. Twothirds of E. coli $(56 / 84,66.7 \%)$ and two-fifths of K. pneumoniae (6/14, 42.9\%) were ESBL-positive (cases, 62) and rest 36 patients were controls (UTI due to non-ESBL E. coli or K. pneumoniae). There was no significant difference in relation to age $(p=0.757)$, sex $(p=0.548)$, presence $(p=0.696)$, duration $(p=0.050)$ or control of diabetes $(p=0.448)$ between cases and controls. Regarding risk factors responsible for UTI due to ESBLpositive organisms, long duration ( $\geq 5$ years) of diabetes was significant (OR=6.87, 95\% CI=2.34-20.16, $p=0.0004)$. On multivariate logistic regression, presence $(p=0.002)$ and long duration of diabetes $(p=0.002)$, past history of UTI $(p=0.004)$ and history of hospitalization due to UTI $(p=0.005)$ appeared as significant risk factors for UTI due to ESBL-positive E. coli or K. pneumoniae. Imipenem, amikacin, gentamycin and nitrofurantoin were among the most sensitive antibiotics.

Conclusion: Almost two-thirds of the UTI cases were due to ESBL-positive organisms in this study. Imipenem, aminoglycosides and nitrofurantoin were among the most sensitive antibiotics. Long duration of diabetes, past history of UTI and hospitalization due to UTI were significant risk factors for ESBL-positivity.
\end{abstract}

Key words: antibiotic sensitivity, extended-spectrum beta-lactamase, frequency, prevalence, risk factor, urinary tract infection.

(BIRDEM Med J 2017; 7(2): 155-159)

\section{Author information}

a. Dr. Muhammad Abdur Rahim, Tabassum Samad, Assistant Professor, Nephrology, BIRDEM General Hospital, Dhaka, Bangladesh.

b. Dr. Palash Mitra, Assistant Registrar, Nephrology, BIRDEM General Hospital, Dhaka, Bangladesh.

c. Dr. Shahana Zaman, Registrar, Cardiology, NICVD, Dhaka, Bangladesh.

d. Samira Humaira Habib, Principal Research Officer, Health Economics Unit, BADAS, Dhaka, Bangladesh.

e. Dr. Samira Rahat Afroze, Registrar, Internal Medicine, BIRDEM General Hospital, Dhaka, Bangladesh.

f. Dr. Wasim Md. Mohosin Ul Haque, Associate Professor, Nephrology, BIRDEM General Hospital, Dhaka, Bangladesh.

g. Prof. Khwaja Nazim Uddin, Professor, Internal Medicine, BIRDEM General Hospital, Dhaka, Bangladesh.

Address of correspondence: Dr. Muhammad Abdur Rahim, Assistant Professor, Nephrology, BIRDEM General Hospital, Dhaka, Bangladesh. Email: muradrahim23@yahoo.com

Received: August 22, $2016 \quad$ Accepted: February 28, 2017

\section{Introduction}

Urinary tract infection (UTI) is one of the most common bacterial infections in adults. Antibiotics remain the cornerstone for management of UTI. Empiric and nonjudicious use of antibiotics lead to resistant strains of bacteria. Extended-spectrum beta-lactamase (ESBL) producing strains are an ever increasing problem throughout the world. ${ }^{1-4}$ In Bangladesh, only a few studies were reported on ESBL-positive organisms, mostly describing their prevalence and antibiotic sensitivity patterns. ${ }^{5-9}$ Diabetes mellitus (DM), past history of UTI and prior antibiotic use are reported risk factors for UTI due to ESBL-positive organisms. ${ }^{10,11}$ In this study, we have tried to evaluate the frequency of UTI due to ESBL-positive Escherichia coli and Klebsiella pneumoniae, their antibiotic sensitivity patterns and risk factors. 


\section{Methods}

This case-control study was done in the Department of Internal Medicine of Bangladesh Institute of Research and Rehabilitation in Diabetes, Endocrine and Metabolic Disorders (BIRDEM) General Hospital from January to March 2016. BIRDEM General Hospital is a 700bed tertiary care hospital in Dhaka, the capital city of Bangladesh. This hospital was initially established and dedicated for serving diabetic patients, later converted into a general hospital and now serving as teaching hospital for BIRDEM Academy and Ibrahim Medical College.

Hospitalized patients with a clinical diagnosis of UTI were initially enrolled for the study purpose. UTI cases were clinically diagnosed depending upon clinical features later confirmed by positive urine cultures. A clean catch technique was applied for urine (preferably early morning midstream specimen) collection. Collected samples were then sent to the microbiology laboratory of the institute within half an hour where these samples were inoculated in MacConkey agar and blood agar media within two hours. Significant culture positive cases (semi-quantitative colony count $>1 \times 10^{5}$ colony forming units $/ \mathrm{ml}$ ) were then included for the study purpose. Catheterized patients, pregnant ladies, patients with inadequate/no growth or growth of candida on urine culture were excluded from the study. Growth of organisms was further evaluated by their colony characters and biochemical tests including triple sugar iron (TSI), motility indole urea (MIU) and Simon citrate. Then the selected samples were sub-cultured for antibiotic sensitivity in Muellar-Hilton (MH) agar by Kibry-Bauer disc susceptibility test. ${ }^{12}$ ESBL-positivity was determined phenotypically by Double Disc Synergy Test. ${ }^{13}$ Finally patients with UTI due to ESBL-positive E. coli and K. pneumoniae were selected as cases and those with non-ESBL organisms were taken as controls.

Selected study participants were then further evaluated by history regarding risk factors including presence of and if yes, then duration of diabetes, history of UTI within last one year, history of antibiotic intake within last one year and history of hospitalization due to UTI within last one year and their laboratory reports like glycated haemoglobin $(\mathrm{HbA} 1 \mathrm{c})$ were recorded. Data were collected in semistructured case record forms. Data were analyzed by statistical package for social sciences (SPSS) version 20.0. Comparison of qualitative and quantitative variables between cases and controls were made by appropriate tests and a $p$ value of $<0.05$ was considered as significant.

\section{Results}

During the study period, a total of 136 culture proven UTI cases were recorded. E.coli $(84,61.8 \%)$ was the most common organism followed by Klebsiella (14, $10.3 \%$ ). Total 98 cases of UTI due to E. coli and Klebsiella were included in this study. Other less common organisms were Enterobacter spp. (2, 1.5\%), Citrobacter (6, 4.4\%), Pseudomonas (3, 2.2\%), Burkholderia pseudomallei (1,0.7\%), Enterococcus (10, 7.4\%), Acinetobacter (6, 4.4\%), Staphylococcus aureus $(7,5.1 \%)$, meticillin resistant $S$. aureus $(1,0.7 \%)$ and Streptococcus (2, 1.5\%). Two-thirds (56/84, 66.7\%) of $E$. coli and two-fifths $(6 / 14,42.9 \%)$ of Klebsiella were ESBL-positive. Base-line characteristics of the cases (62) [ESBL-positive E. coli (56) and Klebsiella (6)] and controls (36) [Non-ESBL E. coli (28) and Klebsiella (8)] are presented in Table I.

Table I. Base-line characteristics of the study participants $(\mathrm{N}=98)$

\begin{tabular}{lccc} 
Characteristics & Cases (ESBL-positive) & Controls (Non-ESBL) & $p$ value \\
\hline Number & 62 & 36 & 0.510 \\
Mean age (years) & $56.42 \pm 14.84$ & $55.50 \pm 12.91$ & 0.757 \\
Sex (F:M) & $2.3: 1$ & $3: 1$ & 0.548 \\
DM:non-DM & $61: 1$ & $35: 1$ & 0.696 \\
Mean DM duration (years) & $9.36 \pm 5.25$ & $7.14 \pm 5.51$ & 0.050 \\
Mean HbA1c (\%) & $9.06 \pm 1.63$ & $9.38 \pm 2.53$ & 0.448 \\
Past history of UTI & 31 & 16 & 0.596 \\
History of prior antibiotic use & 34 & 17 & 0.465 \\
Past history of hospitalization due to UTI & 29 & 10 & 0.064 \\
Neutrophil leukocytosis & 57 & 34 & 0.645 \\
\hline
\end{tabular}


Fever, dysuria and increased urinary frequency were among the most common presentations. Other features are presemted in Table II.

Long ( $\geq 5$ years) duration of diabetes was significant risk factor for UTI with ESBL-postive organisms among the study subjects (Table III). On multivariate logistic regression analysis, presence of DM ( $p=0.002)$, long duration of DM $(p=0.002)$, past history of UTI $(p=0.004)$ and history of prior hospitalizaton due to UTI within one year $(p=0.005)$ were significant risk factors for ESBL-postivity. Antibiotic sensitivity pattern of the study subjects are shown in Table IV.

Table II. Clinical presentation of the study subjects $(\mathrm{N}=98)$

\begin{tabular}{lccc} 
Clinical feature & Total (98) & Cases (ESBL-positive) (62) & Controls (non-ESBL) (36) \\
\hline Fever & $58(59.2)$ & $35(56.5)$ & $23(63.9)$ \\
Increased frequency & $32(32.7)$ & $17(27.4)$ & $15(41.7)$ \\
Dysuria & $39(39.8)$ & $24(38.7)$ & $15(41.7)$ \\
Loin pain & $32(32.7)$ & $19(30.6)$ & $13(36.1)$ \\
Supra-pubic pain & $21(21.4)$ & $11(17.7)$ & $10(27.8)$ \\
Incontinence & $15(15.3)$ & $9(14.5)$ & $6(16.7)$ \\
Vomiting & $27(27.6)$ & $13(21.0)$ & $14(38.9)$ \\
Asymptomatic & $32(32.7)$ & $23(37.1)$ & $9(25.0)$ \\
\hline
\end{tabular}

*most patients had more than one feature

Table III. Risk factors for UTI with ESBL-positive organisms (N=98)

\begin{tabular}{llccccc} 
Risk Factors & \multicolumn{2}{c}{$\begin{array}{c}\text { Cases (62) } \\
\text { ESBL-positive }\end{array}$} & $\begin{array}{c}\text { Controls (36) } \\
\text { Non-ESBL }\end{array}$ & $\begin{array}{c}\text { Odds } \\
\text { ratio }\end{array}$ & 95\% CI & $p$ value \\
\hline Age (years) & $<55$ & 22 & 15 & 1.29 & $0.55-3.01$ & 0.5431 \\
& $\geq 55$ & 40 & 21 & & & \\
Sex & Male & 19 & 9 & 0.75 & $0.29-1.90$ & 0.5515 \\
& Female & 43 & 27 & & & \\
DM & No & 1 & 1 & 1.74 & $0.10-28.74$ & 0.6977 \\
& Yes & 61 & 35 & & & \\
DM duration (years) & $<5$ & 6 & 15 & 6.87 & $2.34-20.16$ & 0.0004 \\
& $\geq 5$ & 55 & 20 & & & \\
HbA1c (\%) & $<7$ & 3 & 3 & 1.81 & $0.34-9.50$ & 0.4819 \\
\multirow{2}{*}{ History of UTI } & $\geq 7$ & 58 & 32 & & & \\
& No & 28 & 19 & 1.35 & $0.59-3.09$ & 0.4674 \\
History of hospitalization & Yes & 34 & 17 & & & \\
due to UTI & No & 33 & 26 & 2.28 & $0.94-5.52$ & 0.0668 \\
\hline
\end{tabular}


Table IV. Antibiotic sensitivity pattern of the ESBLpositive E. coli and Klebsiella pneumoniae

\begin{tabular}{lcc} 
Antibiotic & ESBL-positive & ESBL-positive \\
& E. coli (56) & K. pneumoniae (6) \\
\hline Amikacin & $52(92.9)$ & $6(100.0)$ \\
Ciprofloxacin & $6(9.7)$ & $3(50.0)$ \\
Co-trimoxazole & $20(35.7)$ & $2(33.3)$ \\
Gentamicin & $46(82.1)$ & $4(66.7)$ \\
Imipenem & $56(100.0)$ & $6(100.0)$ \\
Mecillinum & $34(60.7)$ & $3(50.0)$ \\
Netilmicin & $52(92.9)$ & $6(100.0)$ \\
Nitrofurantoin & $52(92.9)$ & $5(83.3)$ \\
\hline
\end{tabular}

\section{Discussion}

In primary care settings, fluroquinolones and oral cephalosporins remain as the major drugs for treating UTI. High resistance against fluroquinolones in UTI is well described. ${ }^{4,14}$ ESBL-positive organisms are inherently resistant against penicillins and cephalosporins. So, ESBL-positive UTI cases are not benefitted with such empiric treatments, rather contributes to antibiotic resistance. Not only that, ESBL positivity of micro-organisms remains an increasing problem in developed as well as in developing countries. ${ }^{1-6,9,15}$

In the current study, almost two-thirds of UTI cases were due to ESBL-positive organisms. In four different studies done in Bangladesh over the preceding years by Rahman MM et al. ${ }^{5}$, Rahman MR et al. ${ }^{9}$, Asna SMHZ et al. ${ }^{6}$ and Iqbal $\mathrm{S}$ et al. ${ }^{7}$ revealed up to $60 \%$ of all UTI cases were due to ESBL-positive organisms. In different studies in India ${ }^{10}$, Nepal ${ }^{16}$, Iran ${ }^{17}$ and Turkey ${ }^{18}$ up to $48 \%$ of UTI cases were due to ESBL-positive organisms.

Long duration of diabetes, poor glycaemic control, prior UTI and history of hospitalization due to UTI were significant risk factors for ESBL positivity in this study. Risk factors are comparable with different study reports through-out the world. ${ }^{10,16-19}$ Antibiotic sensitivity patterns were also similar. ${ }^{6-10,16-18}$

Current study had some limitations. It was a single center study, comprising small number of study population over a short period of time and most of our study participants were diabetic.

In conclusion, it can be said that ESBL-positive organisms were almost two-thirds of UTI cases studied. Two-thirds of $E$. coli and two-fifths of $K$. pneumoniae were ESBL-positive. Imipenem, aminoglycosides and nitrofurantoin were among the most sensitive antibiotics. Long duration and poor control of diabetes, past history of UTI and hospitalization were significant risk factors for ESBL positivity. It might be recommended that, urine culture should be requested in suspected UTI cases specially if patient is diabetic one and if there is a past history of UTI and all centers should test for ESBL positivity routinely.

Acknowledgement: The authors express their acknowledgement to Lovely Barai, Associate Professor of Microbiology, BIRDEM General Hospital for her assistance in performing this research.

Conflict of interest: None declared.

Declaration: This paper was presented in the 33rd World Congress of Internal Medicine, Bali, Indonesia, 2016.

\section{References}

1. Spadafino JT, Cohen B, Liu J, Larson E. Temporal trends and risk factors for extended-spectrum-beta-lactamase producing Escherichia coli in adults with catheter-associated urinary tractinfection. Antimicrob Res Infect Cont 2014;3:39.

2. Kang C-I, Cha MK, Kim SH, Ko KS, Wi YM, Chung DR, et al. Clinical and Molecular Epidemiology of Community-Onset Bacteremia Caused by Extended-Spectrum Beta-LactamaseProducing Escherichia coli over a 6-Year Period. J Korean Med Sci 2013; 28:998-1004.

3. Fennell J, Vellinga A, Hanahoe B, Morris D, Boyle F, Higgins $\mathrm{F}$, et al. Increasing prevalence of ESBL production among Irish clinical Enterobacteriaceae from 2004 to 2008: an observational study. BMC Infect Disease 2012;12:116-23.

4. Rahman MR, Rahim MA, Afroze SR, Afroz F, Haque HF, Mitra P, et al. Pattern of Bacterial Pathogens Causing Urinary Tract Infection and Their Antibiotic Sensitivity: A Tertiary Care Hospital Experience. Birdem Med J 2015;5(1):20-23.

5. Rahman MM, Haq JA, Hossain MA, Sultana R, Islam F, Islam AH. Prevalence of extended-spectrum beta-lactamaseproducing Escherichia coli and Klebsiella pneumoniae in an urban hospital in Dhaka, Bangladesh. Int J Antimicrob Agents 2004;24(5):508-10.

6. Asna SMZH, Akter S, Rahman MM, Mohammad N, Hafez MA. Frequency and Sensitivity of Extended Spectrum BetaLactamase Positive Organisms in a Secondary and Tertiary Level Hospital Netwark in Dhaka. J Enam Med Coll 2015;5(2):80-87. 
7. Iqbal S, Rahim MA, Samad T, Ananna MA, Mitra P, Chowdhury TA. Extended-Spectrum Beta-Lactamase Producing Escherichia coli and Klebsiella pneumoniae are Emerging as Major Pathogens Responsible for Urinary Tract Infection. Bangladesh Crit Care J 2015;3(2):49-52.

8. Masud MR, Afroz H, Fakruddim M. Prevalence of extendedspectrum beta-lactamase positive bacteria in radiologically positive urinary tract infection. Springer Plus 2014;3:216.

9. Rahman MR, Rahim MA, Afroze SR, Poddar CS, Uddin KN. Frequency of Extended-Spectrum Beta-Lactamase Producing Organisms Causing Urinary Tract Infection and Their Antibiotic Sensitivity Pattern. J Medicine 2016;18:3-5.

10. Shaikh S, Fatima J, Shakil S, Rizvi SMD, Kamal MA. Risk factors for acquisition of extended spectrum bata lactamase producing Escherichia coli and Klebsiella pneumoniae in North-Indian hospitals. Saudi J Biol Sci 2015;22:37-41.

11. Ben-Ami R, Rodríguez-Baño J, Arslan H, Pitout JDD, Quentin C, Calbo ES, et al. A Multinational Survey of Risk Factors for Infection with Extended-Spectrum $\beta$-LactamaseProducing Enterobacteriaceae in Nonhospitalized Patients. Clin Infect Dis 2009;49(5):682-90.

12. Bauer AW, Kirby WM, Sherris JC, Turck M. Antibiotic susceptibility testing by a standardized single disk method. Am J Clin Pathol 1966;45(4):493-96.

13. Jarlier V, Nicolas MH, Fournier G, Philippon A. Extended spectrum $\beta$-lactamases conferring transferable resistance to newer $\beta$ - lactam agents in Enterobacteriaceae: Hospital prevalence and susceptibility patterns. Rev Infect Dis 1988; 10:867-78.
14. Mowla R, Imam KMA, Asaduzzaman M, Nasrin N, Raihan SZ, Chowdhury AKA. Emergence of Multidrug Resistant Extended-Spectrum $\beta$-Lactamase Producing Eshcherichia coli Associated With Urinary Tract Infections in Bangladesh. J Basic Clin Pharm 2012;3(1):225-28.

15. Baral P, Neupane S, Marasini BP, Ghimire KR, Lekhak B, Shrestha B. High prevalence of multidrug resistance in bacterial uropathogens from Kathmandu, Nepal. BMC Res Notes 2012;5:38.

16. Chander A, Shrestha D. Prevalence of extended spectrum beta lactamase producing Escherichia coli and Klebsiella pneumoniae urinary isolates in a tertiary care hospital in Kathmandu, Nepal. BMC Res Notes 2013;6:487.

17. Latifpour M, Gholipour A, Damavandi MS. Prevalence of Extended-Spectrum Beta-Lactamase-Producing Klebsiella pneumoniae Isolates in Nosocomial and CommunityAcquired Urinary Tract Infections. Jundishapur J Microbiol 2016; 9(3):e31179.

18. Ozgunes I, Erben N, Kiremitci A, Kartal ED, Durmaz G, Colak $\mathrm{H}$, et al. The prevalence of extended-spectrum beta lactamaseproducing Escherichia coli and Klebsiella pneumoniae in clinical isolates and risk factors. Saudi Med J 2006;27(5):60812.

19. Rubio-Perez I, Martin-Perez E, Garcia DD, Calvo ML-B, Barrera EL. Extended-spectrum beta-lactamase-producing bacteria in a tertiary care hospital in Madrid: epidemiology, risk factors and antimicrobial susceptibility patterns. Emerg Health Threats J 2012;5:11589. 